\title{
African swine fever virus MGF360-11L negatively regulates CGAS-STING-mediated inhibition of type I interferon production
}

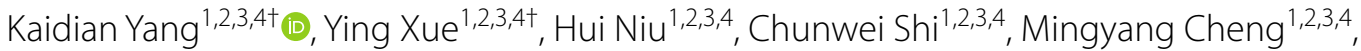

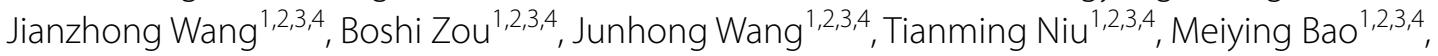 \\ Wentao Yang ${ }^{1,2,3,4}$, Dandan Zhao ${ }^{1,2,3,4}$, Yanlong Jiang ${ }^{1,2,3,4}$, Guilian Yang ${ }^{1,2,3,4}$, Yan Zeng ${ }^{1,2,3,4^{*}}$, Xin Cao $^{1,2,3,4^{*}}$ and \\ Chunfeng Wang ${ }^{1,2,3,4^{*}}$
}

\begin{abstract}
The type I interferon (IFN-I) signaling pathway is an important part of the innate immune response and plays a vital role in controlling and eliminating pathogens. African swine fever virus (ASFV) encodes various proteins to evade the host's natural immunity. However, the molecular mechanism by which the ASFV-encoded proteins inhibit interferon production remains poorly understood. In the present study, ASFV MGF360-11L inhibited CGAS, STING, TBK1, IKKE, IRF7 and IRF3-5D mediated activation of the IFN- $\beta$ and ISRE promoters, accompanied by decreases in IFN- $\beta$, ISG15 and ISG56 mRNA expression. ASFV MGF360-11L interacted with TBK1 and IRF7, degrading TBK1 and IRF7 through the cysteine, ubiquitin-proteasome and autophagy pathways. Moreover, ASFV MGF360-11 L also inhibited the phosphorylation of TBK1 and IRF3 stimulated by CGAS-STING overexpression. Truncation mutation analysis revealed that aa 167-353 of ASFV MGF360-11 L could inhibit cGAS-STING-mediated activation of the IFN- $\beta$ and ISRE promoters. Finally, the results indicated that ASFV MGF360-11L plays a significant role in inhibiting IL-1 $\beta$, IL-6 and IFN- $\beta$ production in PAM cells (PAMs) infected with ASFV. In short, these results demonstrated that ASFV MGF360-11L was involved in regulating IFN-I expression by negatively regulating the CGAS signaling pathway. In summary, this study preliminarily clarified the molecular mechanism by which the ASFV MGF360-11 L protein antagonizes IFN-I-mediated antiviral activity, which will help to provide new strategies for the treatment and prevention of ASF.
\end{abstract}

Keywords: MGF360-11L, IFN-I, signaling pathway, African swine fever virus

\section{Introduction}

The pathogen that causes African swine fever (ASF) is an enveloped, double-stranded DNA virus that exists in the cytoplasm $[1,2]$. ASF has caused serious economic losses to animal husbandry, the meat industry, and foreign trade

\footnotetext{
*Correspondence: zengyan@jlau.edu.cn; inc@jlau.edu.cn; wangchunfeng@jlau.edu.cn

${ }^{\top}$ Kaidian Yang and Ying Xue contributed equally to this paper

${ }^{1}$ College of Veterinary Medicine, Jilin Agricultural University, Changchun, China

Full list of author information is available at the end of the article
}

exports [3-5]. ASF was classified as a category A disease of animals by the World Organization for Animal Health (OIE) and is listed as a category I disease of animals by China [6]. African swine fever virus (ASFV) can evade the host's defense system to combat the innate immune response through a complex interaction between the virus and the host $[4,7,8]$. There are currently no commercially available vaccines or drugs for the prevention and treatment of ASF [4]. Previous studies have reported that ASFV-encoded proteins could effectively inhibit cell and host defenses, which are essential for establishing immune evasion [9]. 
Evasion of host innate immunity plays a significant role in the pathogenesis of ASFV [7]. The innate immune response is the first line of defense against the invasion of microbial pathogens. After cells are infected with a DNA virus, a cytoplasmic sensor detects the viral DNA [10, 11]. Although a variety of cytoplasmic DNA sensors have been identified, cyclic GMP-AMP synthase (cGAS) has been widely accepted for detecting cytoplasmic DNA in a variety of cell types [12-14]. After viral DNA is detected, cGAS catalyzes the synthesis of the second messenger GMP-AMP (cGAMP), which is bound to stimulator of interferon gene (STING), a type I interferon (IFN-I) that is triggered by the virus and is a key adaptor for inducing of the innate antiviral response [15, 16]. STING is activated and translocates to the endoplasmic reticulum (ER), and then tank-binding kinase 1 (TBK1) kinase and the transcription factor interferon response factor 3 (IRF3) are recruited to promote the phosphorylation and dimerization of IRF3 in the ER. Finally, the expression of IFN-I and inflammatory factors is induced by IRF3 in the nucleus to activate the innate immune response $[8,17]$.

A recent study reported that China 2018/1 ASFV DP96R could inhibit the cGAS-STING-TBK1 signaling pathway [18]. ASFV multigene family (MGF) 360-12L blocked the interaction of importin a and the NF- $\mathrm{kB}$ signaling pathway to inhibit IFN-I production [12]. Moreover, ASFV MGF505-7R inhibited the cGAS-STING signaling pathway [19]. pMGF505-7R determines the pathogenicity of ASFV infection by inhibiting IL- $1 \beta$ and IFN-I production [20]. The molecular mechanism by which the immune escape protein of African swine fever virus evades the host's defense system is still poorly understood [12]. Hence, in the present study, we explored the mechanism by which the ASFV immunosuppressive protein MGF360-11L regulates the cGAS-STING signaling pathway. We discovered that ASFV MGF360-11L plays a significant role in inhibiting the IFN-I signaling pathway. To the best of our knowledge, this is the first report to show that ASFV MGF360-11L inhibits IFN-I production by regulating the cGAS-STING signaling pathway. ASFV MGF360-11L interacted with TBK1 and IRF7 and inhibited IFN-I production. These findings could provide a reference for further elucidation of viral immune evasion mechanisms and the development of safe and effective vaccines to prevent ASF.

\section{Materials and methods \\ Cells and viruses}

Porcine kidney (PK)-15, 3D4/21 and human embryonic kidney (HEK)-293 T cells were purchased from the American Type Culture Collection (ATCC) and cultured in RPMI-1640 (Gibco, USA) medium containing 10\% fetal bovine serum (FBS; Gibco) in $5 \% \mathrm{CO}_{2}$ at $37{ }^{\circ} \mathrm{C}$. To
Table 1 The primer sequences for RT-PCR.

\begin{tabular}{ll}
\hline Primers & Sequence $\left(\mathbf{5}^{\prime} \rightarrow \mathbf{3}^{\prime}\right)$ \\
\hline Human IFN- $\beta$-forward & GCTTGGATTCCTACAAAGAAGCA \\
Human IFN- $\beta$-reverse & ATAGATGGTCAATGCGGCGTC \\
Human ISG15-forward & CGCAGATCACCCAGAAGATCG \\
Human ISG15-reverse & TTCGTCGCATTTGTCCACCA \\
Human ISG56-forward & TTGATGACGATGAAATGCCTGA \\
Human ISG56-reverse & CAGGTCACCAGACTCCTCAC \\
Human GAPDH-forward & GGAGCGAGATCCCTCCAAAAT \\
Human GAPDH-reverse & GGCTGTTGTCATACTTCTCATGG \\
Pig IFN- $\beta$-forward & GCTAACAAGTGCATCCTCCAAA \\
Pig IFN- $\beta$-reverse & AGCACATCATAGCTCATGGAAAGA \\
Pig ISG15-forward & GATCGGTGTGCCTGCCTTC \\
Pig ISG15-reverse & CGTTGCTGCGACCCTTGT \\
Pig ISG56-forward & AAATGAATGAAGCCCTGGAGTATT \\
Pig ISG56-reverse & AGGGATCAAGTCCCACAGATTT \\
Pig P72-forward & CCCAGGRGATAAAATGACTG \\
Pig P72-reverse & CACTRGTTCCCTCCACCGATA \\
Pig GAPDH-forward & ACATGGCCTCCAAGGAGTAAGA \\
Pig GAPDH-reverse & GATCGAGTTGGGGCTGTGACT \\
Pig MGF360-11L-forward & GCGGTGGACTATGACCTCAAAGATG \\
Pig MGF360-11L-reverse & TGCGGACCCTTTCTATTTCGTACAG \\
\hline
\end{tabular}

prepare PAM cells (PAMs) as described by Li et al. [21], PAMs were isolated from the lung lavage fluid of specific pathogen-free (SPF) piglets aged 4 weeks. The cells were cultured in complete RPMI- 1640 at $37{ }^{\circ} \mathrm{C}$ with $5 \% \mathrm{CO}_{2}$. Herpes simplex virus (HSV) was stored in our laboratory. ASFV (SY18 strain, GenBank: MH766894) was isolated from pigs as previously described [22].

\section{Fifty percent hemadsorption dose (HAD50) assay}

ASFV was quantified by HAD50 assays according to previously described methods [22]. Briefly, PAMs were seeded in a 96-well plate and infected with tenfold serial dilutions of virus. At 7 days after infection, the HAD50 was determined using the Reed-Muench method. All data are shown as the means of three independent experiments.

\section{Antibodies and reagents Antibodies}

Anti-GAPDH antibodies, anti-Flag agarose affinity gels, and anti-HA agarose affinity gels were supplied by Sigma-Aldrich (Shanghai, China). Mouse anti-Flaghorseradish peroxidase (HRP), anti-HA-HRP and anti-Myc-HRP antibodies were purchased from Roche (Basel, Switzerland). HRP-conjugated goat anti-mouse IgG $(\mathrm{H}+\mathrm{L})$ was obtained from Proteintech Group, Inc. (Rosemont, IL, USA). 


\section{Reagents}

The double-luciferase reporter assay kit was provided by Promega (Madison, USA). The plasmid prep purification kit was purchased from Omega (Georgia, USA). The MiniBEST universal RNA extraction kit, SYBR green qPCR mix and PrimeScript ${ }^{\mathrm{TM}}$ RT reagent kit with gDNA eraser were purchased from TaKaRa (Dalian, China). Lipofectamine 3000 was supplied by Thermo Fisher (Grand Island, USA). Radioimmunoprecipitation assay (RIPA) buffer, DMSO, MG132, and 3-methyladenine (3-MA) were provided by Sigma-Aldrich (Shanghai, China). Polyvinylidene fluoride (PVDF) membranes were purchased from Merck Millipore (Burlington, USA). Bovine serum albumin (BSA) and $5 \times$ SDS loading sample buffer were purchased from Solarbio (Beijing, China). Swine IFN- $\beta /$ IL-1 $\beta /$ IL-6 commercial ELISA kits were supplied by Thermo Fisher (Grand Island, USA).

\section{Construction and transfection of plasmids}

The MGF360-11L gene of ASFV SY18 (GenBank: MH766894) was synthesized and cloned into the pCMV$N$-HA vector with EcoR I and Not I sites by using standard molecular biology techniques [23]. The ASFV MGF360-11L truncation mutants, including MGF36011L-1 (1-180 aa) and MGF360-11L-2 (167-353 aa), were cloned into the pCMV-N-HA vector. Plasmids for Flagtagged cGAS, STING, TRAF3, TRAF6, TBK1, IKKi, IKKE, IRF3, IRF7, and IRF3-5D, and the IFN- $\beta$-Luc and ISRE-Luc luciferase reporter plasmids, as well as pRL-TK, were described previously $[18,19]$. The plasmid DNA of recombinant bacteria was extracted using a plasmid prep purification kit (Omega). The procedure was performed according to the manufacturer's instructions. Briefly, recombinant bacteria were incubated for $16 \mathrm{~h}$ at $37{ }^{\circ} \mathrm{C}$ with shaking and centrifuged at $10000 \times g$ for $1 \mathrm{~min}$ at room temperature (RT) to collect bacterial pellets. Solution I/RNase, solution II, N3 buffer, ETR binding buffer, ETR wash buffer, HBC buffer, and DNA wash buffer were added, the sample was centrifuged, and the filtered liquid was discarded. Finally, the elution buffer was added and centrifuged to collect the filtered liquid. Plasmids in the filtered liquid were stored at $-20{ }^{\circ} \mathrm{C}$. Purified plasmids were co-transfected with Lipofectamine ${ }^{\mathrm{TM}} 3000$ transfection reagent at a ratio of 2:1 to 3:1 Lipofectamine ${ }^{\mathrm{TM}}$

Table 2 siRNA sequences used in this study.

\begin{tabular}{ll}
\hline Primers & Sequence $\left(\mathbf{5}^{\prime} \rightarrow \mathbf{3}^{\prime}\right)$ \\
\hline siMGF360-11L-forward & CAAAUACUGGUACGCGAUAdTdT \\
siMGF360-11L-reverse & UAUCGCGUACCAGUAUUUGdTT \\
siNC-forward & UUCUCCGAACGUGUCACGUTT \\
siNC-reverse & ACGUGACACGUUCGGAGAATT \\
\hline
\end{tabular}

3000/DNA into HEK-293 T cells and PK-15 cells, respectively, and then the cells were cultured in fresh complete DMEM at $37^{\circ} \mathrm{C}$ for $24 \mathrm{~h}$ for subsequent experiments.

\section{RT-PCR}

Total RNA was extracted from the indicated cells, and $1 \mu \mathrm{g}$ of RNA was reverse transcribed to cDNA with a PrimeScript $^{\mathrm{TM}}$ RT reagent kit. RT-PCR was conducted with $1 \mu \mathrm{L}$ of cDNA as a template using SYBR green qPCR mix (Takara) in an AB 7500 system (Applied Biosystems, USA). GAPDH was used as the endogenous control. Human and pig GAPDH were used for normalization, and the results were calculated using $2^{-\Delta \Delta C T}$ method [24]. All samples were analyzed in triplicate. The primers used in this study are described in Table 1.

\section{Dual-luciferase reporter assays}

The reporter plasmids IFN- $\beta$-Luc (100 ng) and ISRE-Luc $(100 \mathrm{ng})$ were respectively co-transfected with the pRLTK plasmid (10 ng) and the indicated plasmids or empty vector plasmid (pCMV-N-HA) into HEK-293 T cells using Lipofectamine ${ }^{\mathrm{TM}} 3000$ (Thermo Fisher). Twentyfour hours after transfection, the cells were treated with $1 \times$ passive lysis buffer (PLB) (Promega) for $20 \mathrm{~min}$ at RT with shaking and then centrifuged to collect the supernatants, and the activities of firefly and Renilla luciferase were determined using a dual-luciferase reporter assay system (Promega). All experiments were independently repeated at least three times.

\section{Western blotting}

The indicated plasmids were co-transfected into HEK$293 \mathrm{~T}$ cells. At $24 \mathrm{~h}$ post-transfection, the cells were lysed with RIPA buffer. Total cell protein was collected and boiled for $10 \mathrm{~min}, 5 \times$ SDS loading buffer (Solarbio) was added, and the samples were centrifuged $(10000 \times g$, $10 \mathrm{~min}, 4^{\circ} \mathrm{C}$ ). Then, each sample was subjected to SDSPAGE followed by transfer onto PVDF membranes (Merck Millipore). The membranes were blocked with $5 \%$ BSA (Solarbio) for $2 \mathrm{~h}$ at RT and then incubated with a specific primary antibody at $4{ }^{\circ} \mathrm{C}$ for $12 \mathrm{~h}$ with shaking. After being washed with PBST (0.5\% Tween 20$)$, the membranes were incubated with secondary antibodies for $2 \mathrm{~h}$ at RT. After being washed using PBST, protein bands were observed and imaged by an Amersham Imager 600 RGB (GE, Marlborough, USA). The following antibodies were used: mouse anti-HA-HRP (1:1000 dilution), mouse anti-Flag-HRP (1:1000 dilution), mouse anti-Myc-HRP (1:2000 dilution), mouse anti-GAPDH (1:3000 dilution), rabbit anti-IRF3 (1:1000 dilution), rabbit anti-TBK1 (1:1000 dilution), phospho-TBK1 (Ser172) (1:1000 dilution), and phospho-IRF3 (Ser386) (1:1000 dilution). 


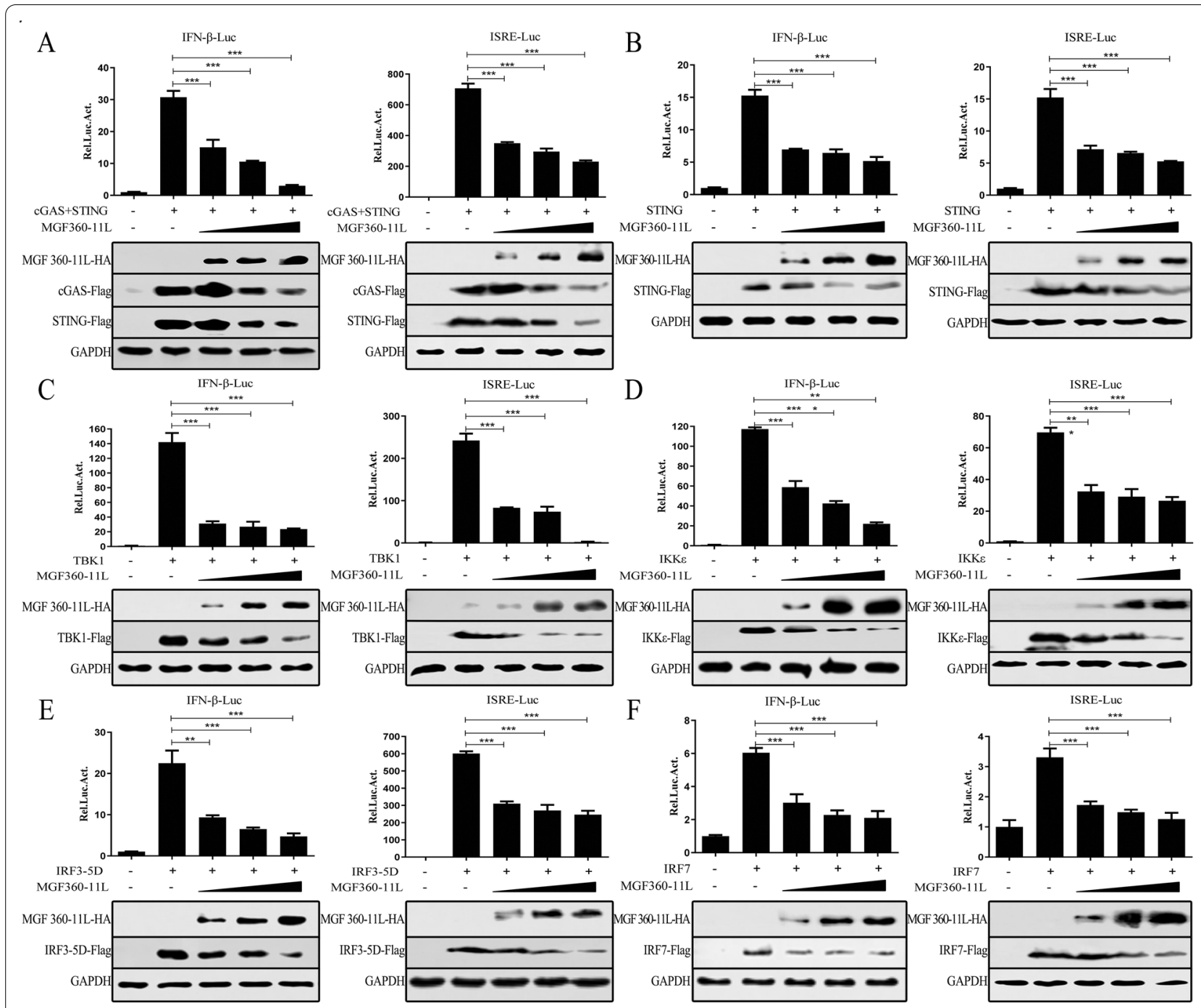

Figure 1 ASFV MGF360-11L inhibited the activation of IFN- $\beta$ and the ISRE promoter. HEK-293 T cells were co-transfected with IFN- $\beta$-LuC (100 ng), ISRE-Luc (100 ng), and pRL-TK (10 ng) plasmids and the IFN-I signaling molecule plasmids CGAS (100 ng, A), STING (100 ng, A), STING (200 ng, B), TBK1 (200 ng, C), IKKE (200 ng, D), IRF3-5D (200 ng, E), and IRF7 (200 ng, F), along with increasing doses of MGF360-11L (50, 100, 200 ng) plasmid or empty vector plasmid ( $\mathrm{pCMV}-\mathrm{N}-\mathrm{HA}$ ). $24 \mathrm{~h}$ post-transfection, cell lysates were used for dual-luciferase reporter assays. The expression of CGAS, STING, TBK1, IKKE, IRF, IRF7 and MGF360-11L was analyzed by Western blotting. All assays were independently repeated at least three times. The data are shown as the mean $\pm \mathrm{SD} ; n=3 .{ }^{*} p<0.05,{ }^{* *} p<0.01,{ }^{* * *} p<0.001$. Luc: luciferase.

\section{Co-immunoprecipitation (Co-IP)}

The indicated plasmids were transfected into HEK-293 T cells. After $24 \mathrm{~h}$ of transfection, the cells were harvested and lysed with IP lysis buffer. For each IP reaction, $1 \mathrm{~mL}$ of lysate was co-incubated with anti-Flag/HA agarose affinity gel (Sigma-Aldrich) for $12 \mathrm{~h}$ at $4{ }^{\circ} \mathrm{C}$ with shaking. After being washed with PBST, the beads were incubated with $1 \mathrm{~mL}$ of lysis buffer and used for the Co-IP assay. Immunoprecipitation was followed by Western blotting with anti-Flag and anti-HA antibodies.

\section{siMGF360-11L-mediated knockdown}

The primers for MGF360-11L siRNAs are listed in Table 2. siRNA transfection was conducted using jetPEI ${ }^{\circledR}$-macrophage in vitro DNA transfection reagent (Polyplus) according to the instructions. SiMGF360-11L was transfected into PAMs for $24 \mathrm{~h}$, and the cells were infected with ASFV $(\mathrm{MOI}=1)$ for another $12 \mathrm{~h}$ or $24 \mathrm{~h}$. IFN- $\beta$, ISG15 and ISG56 mRNA levels were measured by RT-PCR. The cytokines IFN- $\beta$, IL- $1 \beta$ and IL- 6 in the cell culture supernatants were measured by commercial ELISA kits (Thermo Fisher). RT-PCR and Western 


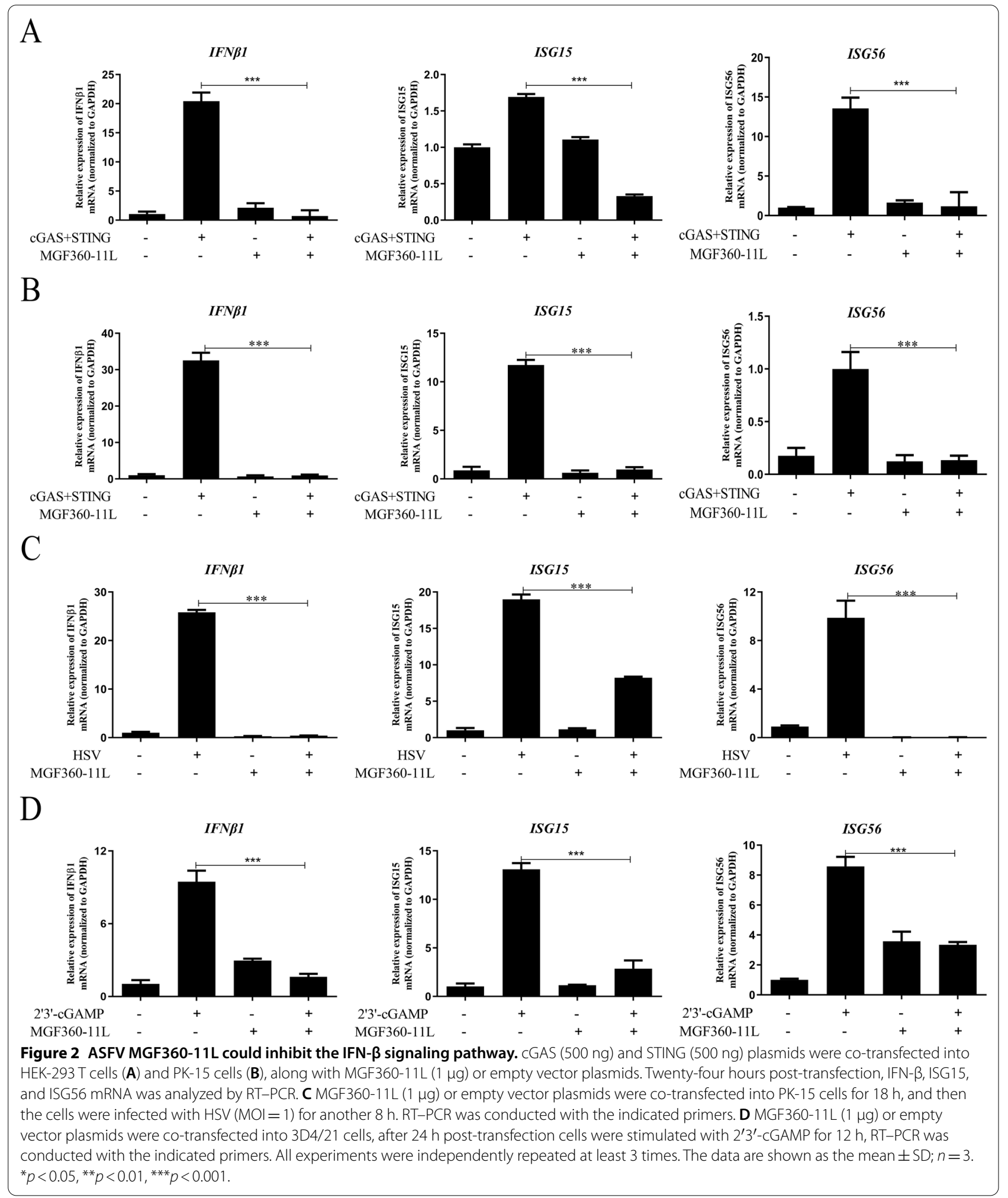

blotting was used to measure the expression level of the P72 protein.

\section{Statistical analysis}

The data in the present study were processed with GraphPad Prism 8 software, and the results are expressed as the 


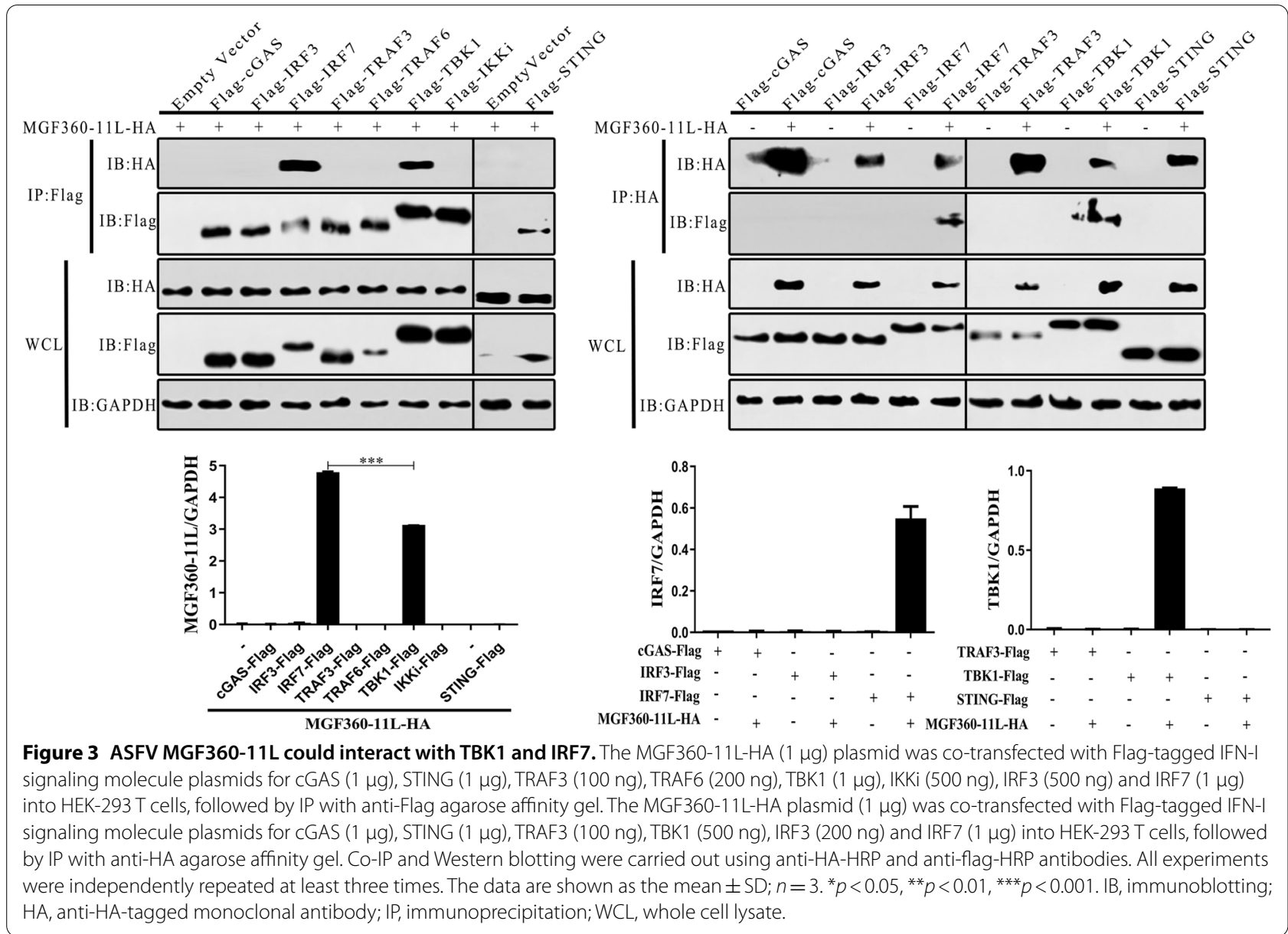

arithmetic means \pm standard deviation. Intragroup or intergroup differences were analyzed by Student's $t$ test or one-way ANOVA, with at least three independent trials. ${ }^{*} P<0.05,{ }^{* *} P<0.01$, and ${ }^{* * *} P<0.001$ were defined as statistically significant.

\section{Results}

ASFV MGF360-11L inhibits activation of the IFN- $\beta$ and ISRE promoters

To explore whether ASFV MGF360-11L could regulate factors in the cGAS-STING signaling pathway, we evaluated the effect of ASFV MGF360-11L on cGAS, STING, TBK1, IRF3-5D (an active form of IRF3), IKKe (a constitutively active form of IKKi) and IRF7 expression by dual-luciferase reporter assays. The results revealed that ASFV MGF360-11L inhibited IFN- $\beta$ and ISRE activation because MGF360-11L inhibited the expression of cGAS/STING (Figure 1A), STING (Figure 1B), TBK1 (Figure 1C), IRF3-5D (Figure 1D), IKKe (Figure 1E) and IRF7 (Figure 1F) in a dose-dependent manner in HEK$293 \mathrm{~T}$ cells, indicating that ASFV MGF360-11L efficiently inhibited the IFN-I response by regulating the cGASSTING pathway.

\section{Effect of ASFV MGF360-11L on the transcription of IFN- $\beta$ and ISGs}

We examined the effect of ASFV MGF360-11L on the inhibition of the IFN-I downstream antiviral response. The results showed that ASFV MGF360-11L could inhibit cGAS-STING-induced transcription of the IFN- $\beta$, ISG15, and ISG56 genes in HEK-293 T cells (Figure 2A). Similarly, ASFV MGF360-11L also inhibited the transcription of the IFN- $\beta$, ISG15, and ISG56 genes triggered by cGAS-STING in PK-15 cells (Figure 2B). Further analysis indicated that in PK-15 cells infected with HSV, ASFV MGF360-11L inhibited transcription of the IFN- $\beta$, ISG15 and ISG56 genes (Figure 2C). ASFV MGF360-11L inhibited transcription of the IFN- $\beta$, ISG15 and ISG56 genes in 3D4/21 cells when stimulated with $2^{\prime} 3^{\prime}$-cGAMP (Figure 2D). These results revealed that ASFV MGF360$11 \mathrm{~L}$ could inhibit the antiviral response of IFN-I downstream genes. 

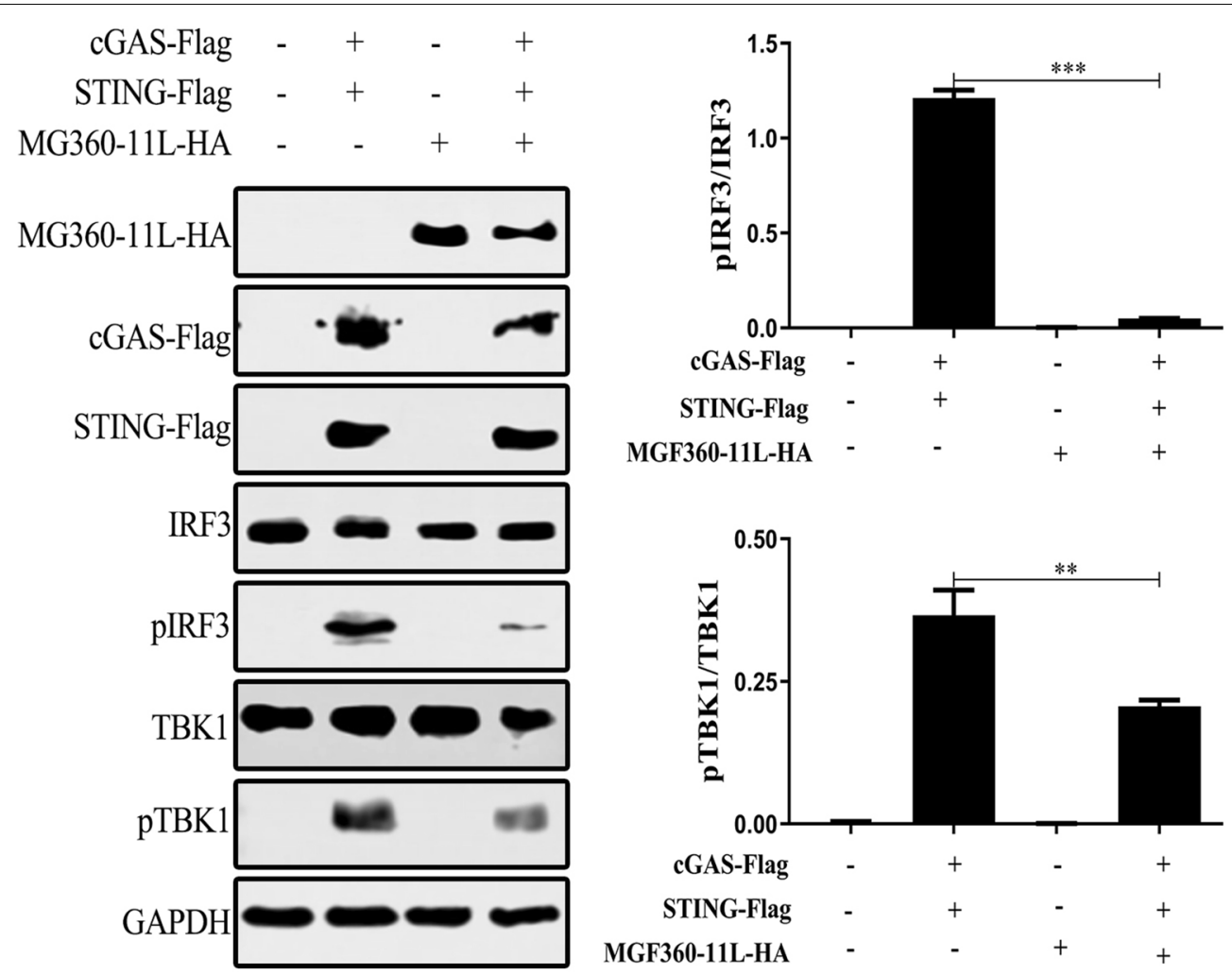

Figure 4 ASFV MGF360-11L inhibited CGAS-STING-induced TBK1 and IRF3 phosphorylation. The MGF360-11L (1 $\mu \mathrm{g})$ plasmid was co-transfected with cGAS (500 ng) and STING (500 ng) plasmids into HEK-293 T cells. At $24 \mathrm{~h}$ post-transfection, the cells were lysed with RIPA buffer, and the phosphorylation of IRF3 and TBK1 was measured by Western blotting. All experiments were independently repeated at least 3 times. The data are shown as the mean $\pm \mathrm{SD} ; n=3 .{ }^{*} p<0.05,{ }^{* *} p<0.01,{ }^{* * *} p<0.001$.

\section{ASFV MGF360-11L interacts with TBK1, IRF7}

To explore the mechanisms of ASFV MGF360-11L in the innate immune response, changes in cGAS, STING, TRAF3, TRAF6, TBK1, IKKi, IRF3 and IRF7 were investigated in response to MGF360-11L. However, it is unclear which molecule in the IFN-I pathway might interact with ASFV MGF360-11L to exert its biological effects. Hence, cGAS, STING, TRAF3, TRAF6, TBK1, IKKi, IRF3 and IRF7 plasmids were co-transfected with MGF360-11L plasmids into HEK-293T cells, respectively (Figure 3). The Co-IP results confirmed that TBK1 and IRF7 could interact with MGF360-11L to inhibit IFN-I expression.

\section{ASFV MGF360-11L inhibits the phosphorylation of TBK1 and IRF3}

TBK1 and IRF3 are vital transcription factors for IFN-I production. The phosphorylation of IRF3 and TBK1 is a hallmark of IRF3 and TBK1 activation. To determine whether ASFV MGF360-11L suppressed IFN-I production by phosphorylating TBK1 or IRF3, plasmids encoding cGAS and STING were co-transfected with the MGF360-11L plasmid into HEK-293T cells, and the results revealed that ASFV MGF360-11L could block the phosphorylation of TBK1 and IRF3 stimulated by cGAS-STING overexpression (Figure 4). These results suggested that ASFV MGF360-11L suppressed the IFN-I immune response by reducing the phosphorylation of TBK1 and inhibiting the downstream activation of IRF3.

\section{ASFV MGF360-11L degrades TBK1 and IRF7}

Experimental evidence has demonstrated that ASFV MGF360-11L can interact with TBK1 and IRF7 (Figures $3 \mathrm{~A}$ and $\mathrm{B})$. We were interested in whether ASFV MGF360-11L could affect the expression of TBK1 and IRF7. TBK1 and IRF7 plasmids were transfected with the ASFV MGF360-11L plasmid into HEK-293 T cells. Western blot analysis revealed that the overexpression of ASFV MGF360-11L led to decreased TBK1 and IRF7 expression (Figures $5 \mathrm{~A}$ and $\mathrm{B}$ ), indicating that ASFV MGF360-11L could degrade TBK1 and IRF7.

To further explore the mechanism by which ASFV MGF360-11L affected the stability of TBK1 and IRF7, HEK-293 T cells were co-transfected with the indicated plasmids for $24 \mathrm{~h}$ and treated with various inhibitors of the protein degradation pathway (Figures $5 \mathrm{C}-\mathrm{F}$ ). We found that ASFV MGF360-11L-induced TBK1 and IRF7 


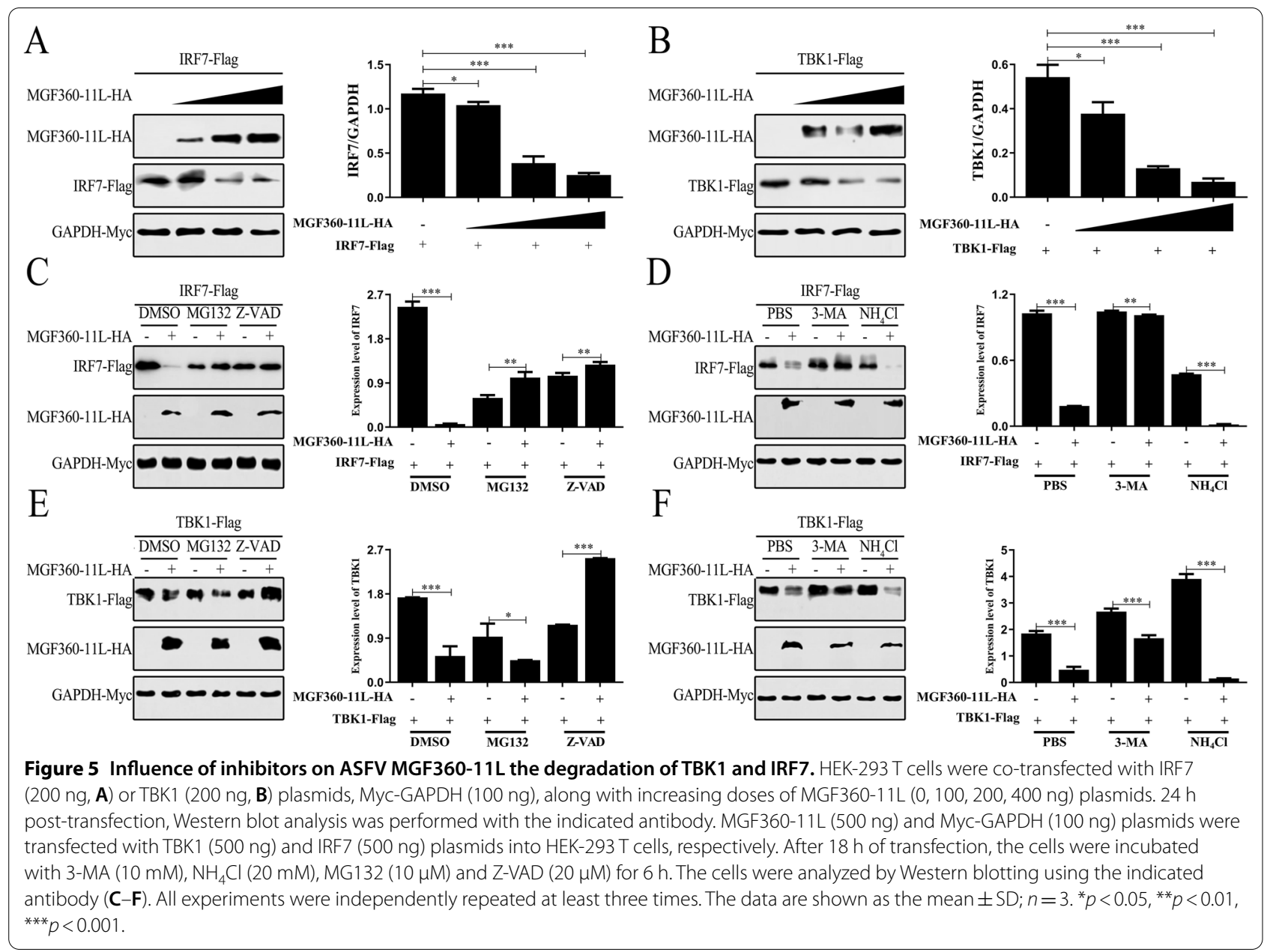

degradation was inhibited by the autophagosome inhibitor 3-MA, cysteine inhibitor Z-VAD and proteasome inhibitor MG132. These results suggested that ASFV MGF360-11L could inhibit the IFN-I signaling pathway by degrading TBK1 and IRF7.

\section{The domains responsible for ASFV MGF360-11L inhibitory activity}

To further determine the domains responsible for ASFV MGF360-11L-mediated inhibitory activity, the ASFV MGF360-11L functional domain was truncated into two segments: MGF360-11L-1 (1-180 aa) and MGF360-11L-2 (167-353 aa). cGAS and STING plasmids were co-transfected with the truncated mutants of ASFV MGF360-11L into HEK-293T cells and examined by dual-luciferase reporter assays and Western blotting. The results indicated that ASFV MGF360-11L and MGF360-11L-2 could inhibit activation of the IFN- $\beta$ promoter by cGAS-STING in HEK-293T cells (Figure 6A). In addition, we also found that the interaction of ASFV MGF360-11L and MGF
360-11L-2 with TBK1 and IRF7 decreased the expression of TBK1 and IRF7 (Figures 6B and C). ASFV MGF36011L-2 inhibited cGAS-STING-induced transcription of the IFN- $\beta$, ISG15 and ISG56 genes in HEK-293 T cells (Figure 6D). ASFV MGF360-11L and MGF360-11L-2 inhibited the IFN-I response, which was related to TBK1 and IRF7.

\section{ASFV MGF360-11L inhibits the expression of IL-1 $\beta$, IL-6 and IFN- $\beta$ in PAMs infected with ASFV}

To verify the effect of ASFV MGF360-11L on inhibiting the IFN-I antiviral response, specific siRNAs against MGF360-11L and nontargeting control siRNA were synthesized and transfected into PAMs, and the cells were infected with ASFV at an MOI of 1.0 for $12 \mathrm{~h}$ or $24 \mathrm{~h}$. The results showed that ASFV MGF360-11L could inhibit the transcription of IFN- $\beta$, ISG15 and ISG56 in PAMs after ASFV infection (Figure 7A), indicating that ASFV MGF360-11L could inhibit the IFN-I antiviral response, which was consistent with our previous study (Figure 2). The IFN- $\beta$, IL-1 $\beta$ and IL-6 levels in cell culture 


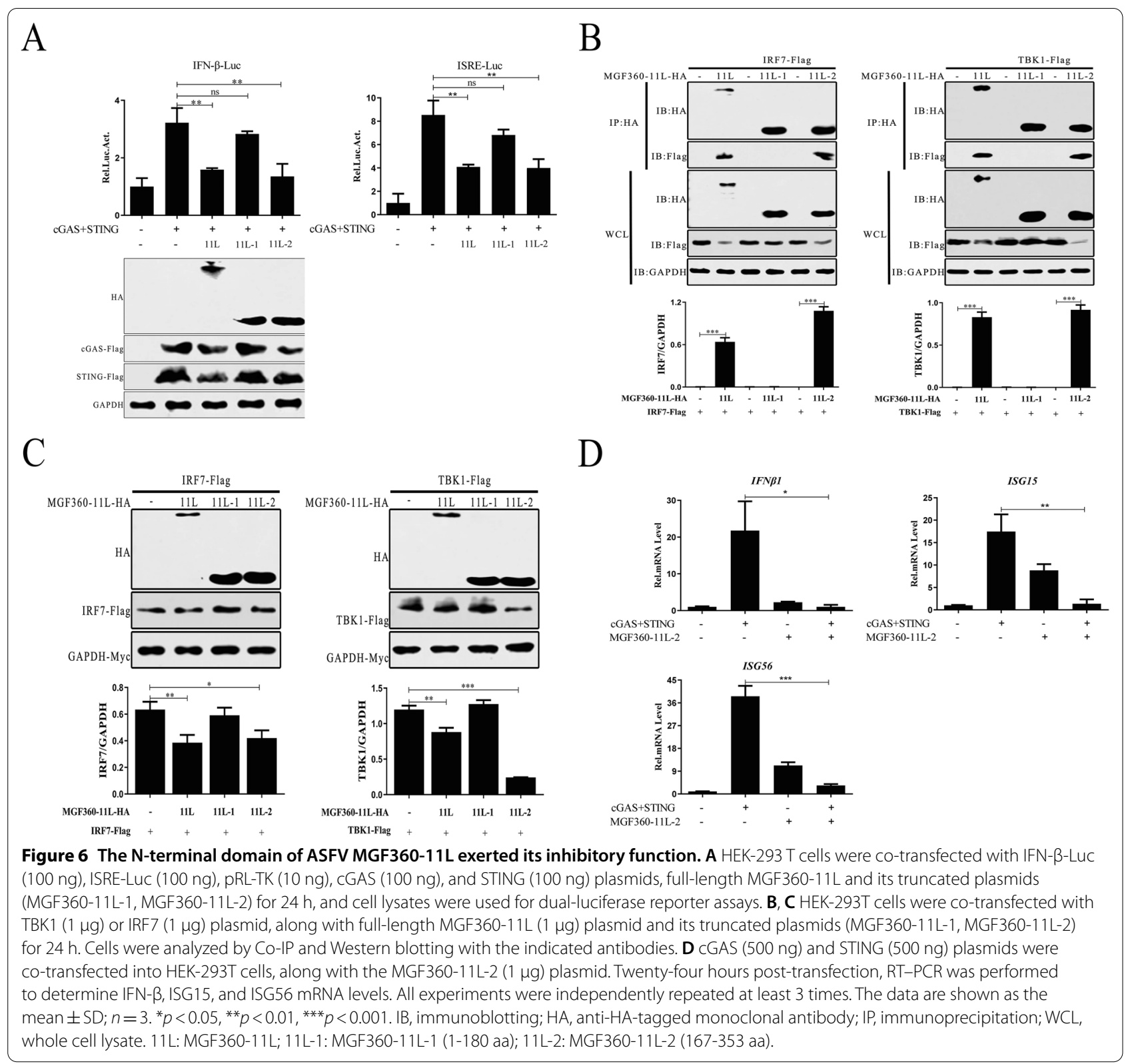

supernatants were measured by ELISA. The results indicated that ASFV MGF360-11L could inhibit the expression of IFN- $\beta$, IL- $1 \beta$ and IL-6 in ASFV-infected PAMs (Figure 7B). Further analysis revealed that compared with nontargeting control siRNA, siMGF360-11L inhibited P72 protein expression in ASFV-infected PAMs (Figure 7C).

\section{Discussion}

ASF is a viral hemorrhagic infectious disease of pigs and wild boars that is caused by ASFV and has a fatality rate as high as $100 \%[25,26]$. There is currently a lack of effective vaccines to prevent and control this disease. Evasion of host innate immunity plays a vital role in the pathogenesis of ASFV. Innate immunity is the host's first line of defense against pathogenic microorganisms $[27,28]$. Inflammation and IFN-I are important components of the innate immune response. How ASFV escapes surveillance by the host innate immune system and the relationship between immune escape strategies and the pathogenicity of the virus are poorly understood. Therefore, we investigated whether the coding gene of the ASFV SY18 strain could mediate immune escape by inhibiting the expression of IFN-I mediated 

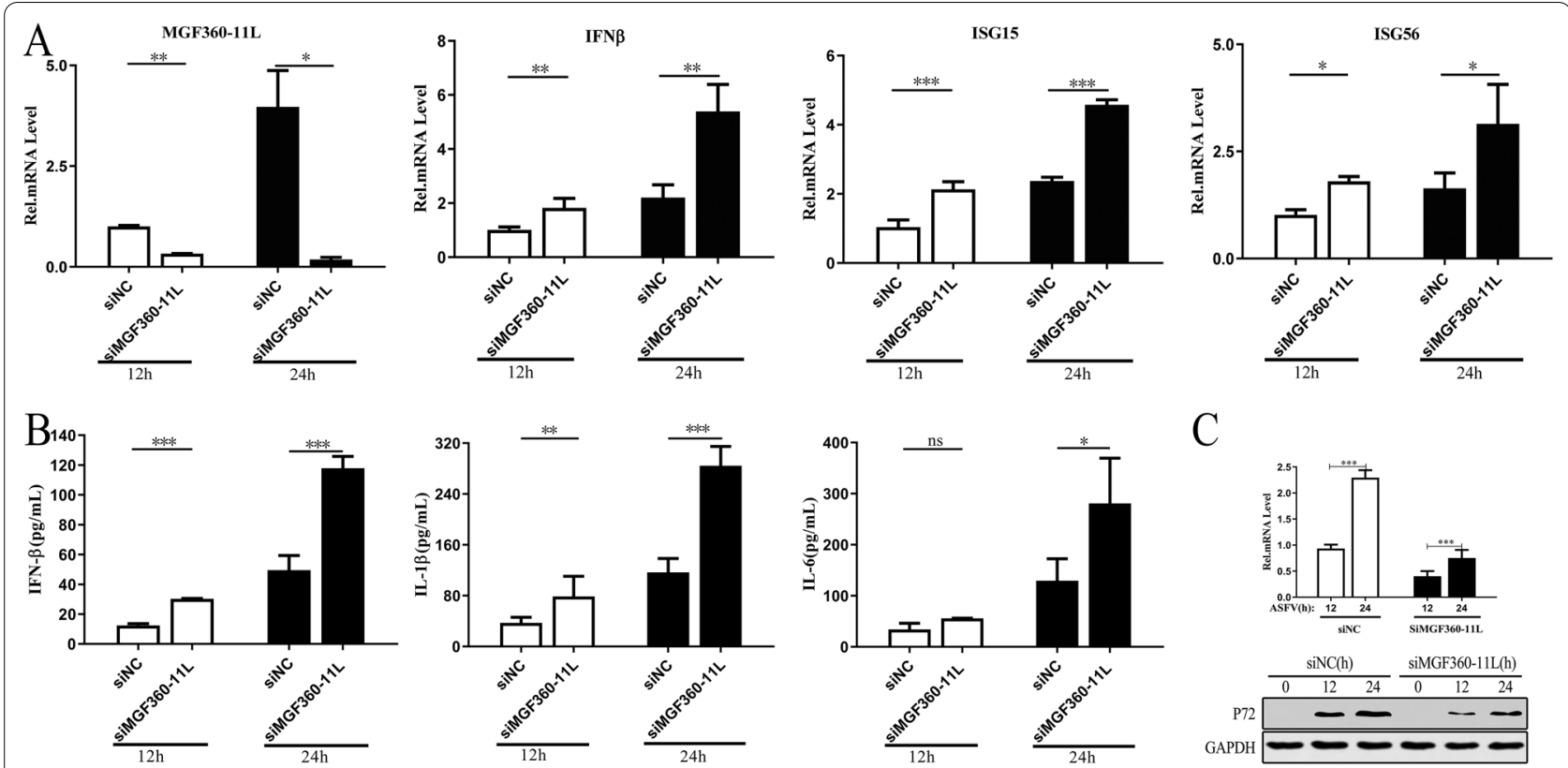

C

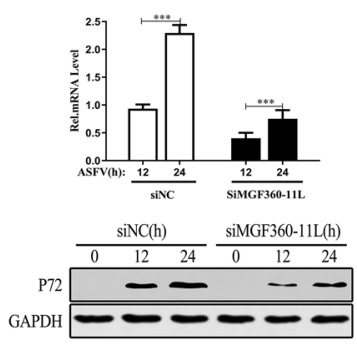

Figure 7 The effect of siMGF360-11 L on the expression of IFN- $\beta$, IL-1 $\beta$ and IL-6. PAMs were transfected with siMGF360-11L or nontargeting control siRNA and infected with ASFV at an MOI of 1.0 for $12 \mathrm{~h}$ or $24 \mathrm{~h}$. A RT-PCR was conducted to measure IFN- $\beta$, ISG15, and ISG56 mRNA levels. B ELISA was carried out to measure the secretion of the cytokines IFN- $\beta$, IL-1 $\beta$ and IL- 6 in the cell supernatant. C RT-PCR and Western blotting were performed to measure the expression level of P72. All experiments were independently repeated at least three times. The data are shown as the mean $\pm S D ; n=3 .{ }^{*} p<0.05,{ }^{* *} p<0.01,{ }^{* * *} p<0.001$.

by cGAS-STING. The genes encoding the ASFV SY18 strain were screened by dual fluorescent reporter genes, and the results revealed that ASFV MGF360-11L has an inhibitory effect on IFN- $\beta$ and ISRE promoter activity (Figures 1A-F).

To evade the host's innate immune response, ASFV encodes many proteins that inhibit the production of IFN-I by antagonizing cGAS-STING signaling. Previous studies have reported that ASFV MGF360 and MGF530/505 play important roles in inhibiting the IFN-I response and antiviral activity [29-31]. ASFV MGF360-11L is a member of ASFV MGF360 and may act as an interferon escape protein to inhibit the host innate immune response. Recent studies have suggested that the ASFV Armenia/07 strain can control the production of IFN- $\beta$ through the cGAS-STING pathway [32]. Additional evidence has shown that China 2018/1 ASFV DP96R could inhibit the production of IFN-I via the cGAS-STING-TBK1 signaling pathway [18]. Moreover, ASFV MGF505-7R could also inhibit the production of IFN-I via the cGAS-STING signaling pathway [19]. In this study, ASFV MGF360-11L not only inhibited cGASSTING-mediated activation of the IFN- $\beta$ and ISRE promoters in a dose-dependent manner in HEK-293T cells but also significantly inhibited downstream IFN-I antiviral responses.
TBK1 is a key transcription factor in the cGAS-mediated signaling pathway, and its phosphorylation is essential for the activation of IRF3 and NF- $\mathrm{kB}$ and induction of IFN- $\beta[33,34]$. IRF-7 is the most important regulator that induces the expression of IFN-I [35]. Studies have shown that the ASFV DP96R protein can suppress the antiviral immune response by reducing the phosphorylation of TBK1 [18]. ASFV MGF505-7R inhibited the phosphorylation of TBK1 and IRF3 induced by B-DNA transfection in PAMs [19]. In the present study, ASFV MGF360-11L interacted with TBK1 and IRF7 and degraded TBK1 and IRF7 through the cysteine, ubiquitin-proteasome and autophagy pathways. Moreover, ASFV MGF360$11 \mathrm{~L}$ could also inhibit the IFN-I response by reducing the phosphorylation of TBK1 and IRF3. Mechanistically, we demonstrated that ASFV MGF360-11L could inhibit IFN-I production by binding to TBK1 and IRF7. Therefore, our results revealed another mechanism by which ASFV escaped host IFN-I signaling by directly targeting TBK1 and IRF7.

Innate immunity is the host's first line of defense against viral infection. When the body is invaded by pathogenic microorganisms, there are a series of pattern recognition receptors (PRRs) in the innate immune system that recognize virus-derived porcine alveolar 
macrophages (PAMs) and trigger a series of signals, causing the production of IFN and the inflammatory cytokines IL-1 $\beta$ and IL- 6 to resist viral infection [36]. Recent studies have shown that pMGF505-7R can interact with NLRP3 to inhibit NLRP3 inflammasome assembly, leading to decreased IL-1 $\beta$ production [20]. Sendai virus V protein can inhibit the secretion of IL-1 $\beta$ by preventing NLRP3 inflammasome assembly [37]. In this study, ASFV MGF360-11L inhibited the production of the inflammatory factors IL- $1 \beta$, IL- 6 and IFN- $\beta$ during ASFV infection. ASFV MGF360-11L can inhibit the translocation and activation of NF- $\mathrm{kB}$, thereby limiting the synthesis of inflammatory factors (IL- $1 \beta$ and IL-6).

In conclusion, our results demonstrated that ASFV MGF360-11L inhibited IFNs and ISGs, blocked p-TBK1 and p-IRF3, interacted with TBK1 and IRF7, and degraded TBK1 and IRF7 by the cysteine and autophagy pathways to inhibit IFN-I-mediated antiviral activity. We will knock out the MGF360-11L gene and construct an ASFV strain with MGF 360-11L gene deletion to obtain safe and effective attenuated ASF vaccine candidate strains. This study provides potential strategies for the development of ASFV attenuated vaccines.

\section{Acknowledgements}

We thank Changchun Veterinary Research Institute, Chinese Academy of Agricultural Sciences.

\section{Authors' contributions \\ K-DY, XC and W-TY designed the study; K-DY, YX, HN, C-WS, D-DZ, T-MN, M-YB and $Y-L J$ were involved in the acquisition of data, analysis, and figure prepara- tion; K-DY, M-YC, J-ZW, J-HW contributed to some of the laboratory experi- ments and data analysis; $Y X$ and B-SZ helped revise the manuscript; G-LY, YZ and C-FW supervised the study; K-DY drafted the original paper. All authors read and approved the final manuscript.}

\section{Funding}

This work was supported by the China Agriculture Research System of MOF and MARA (CARS-35), the National Natural Science Foundation of China (31941018, 81760287, 32072888, 32072897), the Science and Technology Development Program of Jilin Province (YDZJ202102CXJD029, 20190301042NY, 20200402041NC).

\section{Availability of data and materials}

All data generated or analyzed during this study are included in this published article.

\section{Declarations}

\section{Competing interests}

The authors declare that they have no competing interests.

\begin{abstract}
Author details
${ }^{1}$ College of Veterinary Medicine, Jilin Agricultural University, Changchun, China. ${ }^{2}$ Jilin Provincial Key Laboratory of Animal Microecology and Healthy Breeding, Jilin Agricultural University, Changchun, China. ${ }^{3}$ Jilin Provincial Engineering Research Center of Animal Probiotics, Jilin Agricultural University, Changchun, China. ${ }^{4}$ Key Laboratory of Animal Production and Product Quality Safety of Ministry of Education, Jilin Agricultural University, Changchun, China.
\end{abstract}

Received: 8 November 2021 Accepted: 7 January 2022

Published online: 24 January 2022

\section{References}

1. Simoes M, Martins C, Ferreira F (2015) Early intranuclear replication of african swine fever virus genome modifies the landscape of the host cell nucleus. Virus Res 210:1-7. https://doi.org/10.1016/j.virusres.2015.07.006

2. Simoes M, Freitas FB, Leitao A, Martins C, Ferreira F (2019) African swine fever virus replication events and cell nucleus: new insights and perspectives. Virus Res 270:197667. https://doi.org/10.1016/j.virusres.2019.197667

3. Bellini S, Casadei G, De Lorenzi G, Tamba M (2021) A review of risk factors of african swine fever incursion in pig farming within the european union scenario. Pathogens 10:84. https://doi.org/10.3390/pathogens10010084

4. Netherton CL, Connell S, Benfield CTO, Dixon LK (2019) The genetics of life and death: virus-host interactions underpinning resistance to african swine fever, a viral hemorrhagic disease. Front Genet 10:402. https://doi. org/10.3389/fgene.2019.00402

5. Rodriguez JM, Salas ML (2013) African swine fever virus transcription. Virus Res 1739:15-28. https://doi.org/10.1016/j.virusres.2012.09.014

6. Wang FX, Zhang H, Hou LN, Yang C, Wen YJ (2021) Advance of african swine fever virus in recent years. Res Vet Sci 136:535-539. https://doi.org/ 10.1016/j.rvsc.2021.04.004

7. Portugal R, Leitao A, Martins C (2018) Modulation of type I interferon signaling by African swine fever virus (ASFV) of different virulence L60 and NHV in macrophage host cells. Vet Microbiol 216:132-141. https:// doi.org/10.1016/j.vetmic.2018.02.008

8. Dixon LK, Islam M, Nash R, Reis AL (2019) African swine fever virus evasion of host defences. Virus Res 266:25-33. https://doi.org/10.1016/j.virusres. 2019.04.002

9. Wang J, Shi XJ, Sun HW, Chen HJ (2020) Insights into African swine fever virus immunoevasion strategies. J Integr Agr 19:11-22. https://doi.org/10. 1016/S2095-3119(19)62762-0

10. Simoes M, Martins C, Ferreira F (2013) Host DNA damage response facilitates African swine fever virus infection. Vet Microbiol 165:140-147. https://doi.org/10.1016/j.vetmic.2013.01.007

11. Simoes M, Rino J, Pinheiro I, Martins C, Ferreira F (2015) Alterations of nuclear architecture and epigenetic signatures during African swine fever virus infection. Viruses 7:4978-4996. https://doi.org/10.3390/v7092858

12. Zhuo Y, Guo Z, Ba T, Zhang C, He L, Zeng C, Dai H (2021) African swine fever virus MGF360-12L inhibits type I interferon production by blocking the interaction of importin alpha and NF-kappaB signaling pathway. Virol Sin 36:176-186. https://doi.org/10.1007/s12250-020-00304-4

13. Chen Q, Sun LJ, Chen ZJJ (2016) Regulation and function of the CGASSTING pathway of cytosolic DNA sensing. Nat Immunol 17:1142-1149. https://doi.org/10.1038/ni.3558

14. Motwani M, Pesiridis S, Fitzgerald KA (2019) DNA sensing by the cGASSTING pathway in health and disease. Nat Rev Genet 20:657-674. https:// doi.org/10.1038/s41576-019-0151-1

15. Zhang XW, Bai XC, Chen ZJJ (2020) Structures and mechanisms in the cGAS-STING innate immunity pathway. Immunity 53:43-53. https://doi. org/10.1016/j.immuni.2020.05.013

16. Tan XJ, Sun LJ, Chen JQ, Chen ZJJ (2018) Detection of microbial infections through innate immune sensing of nucleic acids. Annu Rev Microbiol 72:447-478. https://doi.org/10.1146/annurev-micro-102215-095605

17. Wu JJ, Dobbs N, Yang K, Yan N (2020) Interferon-independent activities of mammalian STING mediate antiviral response and tumor immune evasion. Immunity 53:115-126.e5. https://doi.org/10.1016/j.immuni.2020.06. 009

18. Wang XX, Wu J, Wu YT, Chen HJ, Zhang SF, Li JX, Xin T, Jia H, Hou SH, Jiang YT, Zhu HF, Guo XY (2018) Inhibition of CGAS-STING-TBK1 signaling pathway by DP96R of ASFV China 2018/1. Biochem Biophys Res Commun 506:437-443. https://doi.org/10.1016/j.bbrc.2018.10.103

19. Li D, Yang W, Li L, Li P, Ma Z, Zhang J, Qi X, Ren J, Ru Y, Niu Q, Liu Z, Liu X, Zheng H (2021) African swine fever virus MGF-505-7R negatively regulates CGAS-STING-mediated signaling pathway. J Immunol 206:18441857. https://doi.org/10.4049/jimmunol.2001110

20. Li J, Song J, Kang L, Huang L, Zhou S, Hu L, Zheng J, Li C, Zhang X, He X, Zhao D, Bu Z, Weng C (2021) pMGF505-7R determines pathogenicity of African swine fever virus infection by inhibiting IL-1 beta and type I IFN production. PLoS Pathog 17:e1009733. https://doi.org/10.1371/journal. ppat.1009733

21. Li J, Hu L, Liu Y, Huang L, Mu Y, Cai X, Weng C (2015) DDX19A senses viral RNA and mediates NLRP3-dependent inflammasome activation. J Immunol 195:5732-5749. https://doi.org/10.4049/jimmunol.1501606 
22. Zhao D, Liu R, Zhang X, Li F, Wang J, Zhang J, Liu X, Wang L, Zhang J, Wu X, Guan Y, Chen W, Wang X, He X, Bu Z (2019) Replication and virulence in pigs of the first African swine fever virus isolated in China. Emerg Microbes Infect 8:438-447. https://doi.org/10.1080/22221751.2019.15901 28

23. Sambrook J, Fritsch EF, Maniatis T (1989) Molecular cloning: a laboratory manual, 2nd edn. Cold Spring Harbor Laboratory Press, New York

24. Livak KJ, Schmittgen TD (2001) Analysis of relative gene expression data using real-time quantitative PCR and the $2^{-\triangle C C T}$ method. Methods 25:402-408. https://doi.org/10.1006/meth.2001.1262

25. Wang T, Sun Y, Huang SJ, Qiu HJ (2020) Multifaceted immune responses to African swine fever virus: implications for vaccine development. Vet Microbiol 249:108832. https://doi.org/10.1016/JVetmic.2020.108832

26. Gao X, Liu T, Liu YX, Xiao JH, Wang HB (2021) Transmission of African swine fever in China through legal trade of live pigs. Transbound Emerg Dis 68:355-360. https://doi.org/10.1111/tbed.1368

27. Reis AL, Netherton C, Dixon LK (2017) Unraveling the armor of a killer: evasion of host defenses by African swine fever virus. J Virol 91:e02338-e2416. https://doi.org/10.1128/JVI.02338-16

28. Teklue T, Sun Y, Abid M, Luo YZ, Qiu HJ (2020) Current status and evolving approaches to African swine fever vaccine development. Transbound Emerg Dis 67:529-542. https://doi.org/10.1111/tbed.13364

29. Malogolovkin A, Kolbasov D (2019) Genetic and antigenic diversity of African swine fever virus. Virus Res 271:197673. https://doi.org/10.1016/j, virusres.2019.197673

30. Ramirez-Medina E, Vuono EA, Velazquez-Salinas L, Silva E, Rai A, Pruitt S, Berggren KA, Zhu J, Borca MV, Gladue DP (2020) The MGF360-16R ORF of African swine fever virus strain Georgia encodes for a nonessential gene that interacts with host proteins SERTAD3 and SDCBP. Viruses 12:60. https://doi.org/10.3390/V12010060

31. Afonso CL, Piccone ME, Zaffuto KM, Neilan J, Kutish GF, Lu Z, Balinsky CA Gibb TR, Bean TJ, Zsak L, Rock DL (2004) African swine fever virus multigene family 360 and 530 genes affect host interferon response. J Virol 78:1858-1864. https://doi.org/10.1128/jvi.78.4.1858-1864.2004

32. Garcia-Belmonte R, Perez-Nunez D, Pittau M, Richt JA, Revilla Y (2019) African swine fever virus Armenia/07 virulent strain controls interferon beta production through the cGAS-STING pathway. J Virol 93:e02298-e2318. https://doi.org/10.1128/JVI.02298-18

33. Correia S, Ventura S, Parkhouse RM (2013) Identification and utility of innate immune system evasion mechanisms of ASFV. Virus Res 173:87-100. https://doi.org/10.1016/j.virusres.2012.10.013

34. Zhong B, Yang Y, Li S, Wang YY, Li Y, Diao FC, Lei CQ, He X, Zhang L, Tien $P$, Shu HB (2008) The adaptor protein MITA links virus-sensing receptors to IRF3 transcription factor activation. Immunity 29:538-550. https://doi. org/10.1016/j.immuni.2008.09.003

35. Honda K, Yanai H, Negishi H, Asagiri M, Sato M, Mizutani T, Shimada N, Ohba Y, Takaoka A, Yoshida N, Taniguchi T (2005) IRF-7 is the master regulator of type-l interferon-dependent immune responses. Nature 434:772-777. https://doi.org/10.1038/nature03464

36. Liu ZY, Qin Q, Wu C, Li H, Shou JN, Yang YT, Gu MD, Ma CM, Lin WL, Zou Y, Zhang YY, Ma F, Sun JH, Wang XJ (2018) Downregulated NDR1 protein kinase inhibits innate immune response by initiating an miR146aSTAT1 feedback loop. Nat Commun 9:2789. https://doi.org/10.1038/ s41467-018-05176-7

37. Komatsu T, Tanaka Y, Kitagawa Y, Koide N, Naiki Y, Morita N, Gotoh B, Yokochi T (2018) Sendai virus $V$ protein inhibits the secretion of interleukin-1 beta by preventing NLRP3 inflammasome assembly. J Virol 92:e00842-e918. https://doi.org/10.1128/JVI.00842-18

\section{Publisher's Note}

Springer Nature remains neutral with regard to jurisdictional claims in published maps and institutional affiliations.

Ready to submit your research? Choose BMC and benefit from:

- fast, convenient online submission

- thorough peer review by experienced researchers in your field

- rapid publication on acceptance

- support for research data, including large and complex data types

- gold Open Access which fosters wider collaboration and increased citations

- maximum visibility for your research: over $100 \mathrm{M}$ website views per year

At BMC, research is always in progress.

Learn more biomedcentral.com/submissions 